\title{
El concepto de la muerte en el imaginario mexicano.
}

\author{
The concept of death in the Mexican imaginary. \\ Sergio J. Villaseñor-Bayardo ${ }^{1,2, a}$, Martha P. Aceves Pulido ${ }^{2, b}$
}

\section{RESUMEN}

Se examina el concepto de la muerte y elementos relacionados en el contexto de la cultura Nahua del antiguo México, antes y después de la conquista española. En términos generales, la muerte no era considerada un evento penoso o angustiante, aun cuando los poetas y artistas cantaban a la caducidad de la vida y las angustias de la agonía. Los mitos enfatizaban trascendencia y re-creación. Las moradas de los fallecidos variaban en función de edad y tipo de muerte. Por ejemplo, un soldado muerto en combate o eliminado como prisionero por sus enemigos, o una mujer que fallecía durante el parto, reposarían en la llamada Casa del Sol. Lactantes fallecidos simplemente retornaban al $13^{\circ}$. cielo, de donde habían venido, a seguir alimentándose del árbol nodriza y aun con posibilidades de regresar a la tierra. Nombres asignados a "lugares de los muertos" incluyen Tlalocan, Mictlan y Tlatilpac. La llegada del cristianismo cambió sustancialmente algunas de estas creencias aun cuando, como en el caso del Día de Difuntos, se estableció una suerte de sincretismo en la asignación de fechas y la práctica de rituales recordatorios.

PALABRASCLAVE: Día de los muertos, cultura nahua, mitos, imaginario cultural.

\section{SUMMARY}

The concept of death and related topics in the context of the Nahua culture from ancient Mexico, before and after the Spanish conquest, are examined. In general, death was not considered an anguishing or painful event, even though poets and artists used to explore the transiency of life and the anxieties of agony. Myths would emphasize transcendence and re-creation. The homes of the dead varied in connection with age and type of death. For example, a soldier who died in the battlefield or was killed when a prisoner of his enemies, or a woman who would die during labor would rest in the so-called House of the Sun; breastfeeding infants would simply return to tre they had come from to continue nourishing from the "wetnurse tree", still with possibilities of returning to Earth. Names assigned to this sites included Tlalocan, Mictlan y Tlatilpac. The arrival of Christianity substantially changed some of these beliefs even though, as was the case with the "Day of the Dead" (Día de Difuntos), a sort of syncretism was established about dates' assignments and the practice of memorial rituals.

KEY WORDS: Day of the dead, Nahua culture, myths, cultural imagery.

\section{INTRODUCCIÓN}

Para comprender cabalmente el concepto actual de la muerte en el imaginario del pueblo mexicano es menester retroceder a épocas pasadas. De la época prehispánica destacan los pueblos nahuas quienes tuvieron un particular pensamiento sobre la vida y la muerte que los llevó a integrarse de una forma excepcional con el devenir del cosmos mismo. $\mathrm{Su}$ concepción cosmológica hace del hombre el centro alrededor del cual gira todo y que da, por lo tanto, sentido a todo. El hombre es responsable, junto con las divinidades de la muerte, de la permanencia del universo total.

Para los antiguos mexicanos la muerte no era angustiante ni amedrentadora, no había por qué rehuirle ni darle la vuelta sino más bien enfrentarla de

\footnotetext{
Presidente-electo, World Association of Cultural Psychiatry. Roma, Italy.

Profesor de la Universidad de Guadalajara. Guadalajara, Jalisco, México

a M.D. Ph.D.; ${ }^{\text {b }}$ Maestra en Psicología.
} 
pie y con entereza. Es algo que si bien no agrada, se acepta con tranquilidad, el vivir es breve, sus poetas -cuicanime- lo saben y lo expresan constantemente: "Tan solo un poco aquí..."

El más allá esperado no es cruel ni condenatorio, no es un lugar de penas ni sufrimientos, por lo cual no hay que temerle. Lo importante es cómo morir pues la trascendencia y la continuidad dependen de esto. No es lo mismo morir de muerte natural que morir de una muerte elegida por los dioses. Tampoco es lo mismo morir de adulto que de pequeño, así como no hay muertes más nobles y gloriosas que la muerte en la xochiyaoyotl (guerra florida) (1), la muerte en el techcatl (piedra de los sacrificios) o la muerte ocasionada por el parto.

Sus ritos funerarios y de duelo reflejaban la sabiduría del pueblo para realizar la catarsis del vacío que deja el muerto con su ausencia y así reanudar la vida con normalidad. Con la invasión española todo quedó truncado, nuevas concepciones fueron impuestas. Un mundo extraño y ajeno impactaba el mundo conocido y propio. Por medio del sincretismo se intentó rescatar lo salvable; en medio de grandes dificultades, presiones y frustraciones, algo consiguió sobrevivir de todo aquello. Hoy, al pasar los siglos, esos elementos sincréticos matizan la tradición y el pensamiento de México.

\section{Mitos nahuas sobre la vida y la muerte}

Llámese alma o energía vital, lo cierto es que los nahuas creían que algo del hombre iba más allá. Ello se comprueba examinando los lugares claramente definidos hacia donde se dirigían los muertos después de fallecer. Ellos demuestran un anhelo profundo de inmortalidad para todos; desde antiguo se pensaba en una inmortalidad en la que también se creía con fervor. El mito de origen, narrado por Sahagún (1), ya contiene tales ideas: "Y se llamaba Teotihuacan, el pueblo de Téotl, que es dios, porque los señores que ahí se enterraban eran canonizados por los dioses después de muertos, no se morían sino que despertaban de un sueño en el que habían vivido; los hombres no perecían, comenzaban a vivir de nuevo ...y se convertían en espíritus o dioses".

Aun cuando la inmortalidad era una forma de trascendencia humana, no por eso dejaron los nahuas de tener ideas aún más amplias y profundas al respecto. En el mito-leyenda de Quetzalcóatl, la serpiente alada junta a la tierra con el cielo y da una medida de lo que nahuas consideraban como trascendencia; sin embargo, el concepto se amplía con el nahualli, el doble de Quetzalcóatl, que es Xólotl, el gemelo, portador del fuego celeste, el que desciende (descender para los nahuas es nacer, es ser) al inframundo, a la región oscura, y no se pierde porque lleva la chispa de luz divina que le permite salir airoso de la lucha que libra en el inframundo y encontrar así el camino para emerger triunfante.

Siguiendo el mito-leyenda de Quetzalcóatl, éste se identifica con el sabio y noble gobernante Tollan (Tula) en su personaje de Ce-ácatl Topiltzin (unocaña, "nuestro príncipe"). Después de abandonar su señorío debido al hostigamiento de los hechiceros, se dirige a Tlillan Tlapallan (lugar donde se juntan el negro y el rojo, imagen metafórica de la que se disfraza la sabiduría) o el quemadero; llegado allí vistió sus insignias de plumas y colocó su máscara, posteriormente se prendió fuego y sus cenizas vieron encumbrarse el corazón de Quetzalcóatl enrumbado al cielo e ingresando a él. Los ancianos afirman que se convirtió en la estrella del alba que apareció como tal la primera vez que apareció; sin embargo, antes de que esto ocurriera, fue a morar entre los muertos, en el mictlan, por cuatro días y aprovechó otros cuatro para proveerse de flechas, por lo cual a los ocho días salió como el lucero y se entronizó como Señor (2). En verdad, puede aceptarse que nada puede ser más tras trascendente que un mortal convertido en planeta y en dios. De aquí se desprende la costumbre de que los tlatoani ("el que manda porque tiene voz") después de su muerte eran quemados con las insignias de Quetzalcóatl, para expresar inequívocamente su deseo de trascender también en una estrella, testimonio de divinización.

Quetzalcóatl (serpiente de plumas de quetzal) es un numen complejo que aglutina una gran diversidad de ideas: como serpiente-pájaro es anuncio de las lluvias fertilizantes, del agua celeste, por lo que está en relación con la tierra y la fertilidad. Es también el dios de la vida, el que después de haber creado al hombre busca cómo alimentarlo y trae el maíz para él. Les enseñó la ciencia, a medir el tiempo, a estudiar los astros y a hacer uso del calendario. Es el arquetipo de la santidad.

El numen más poderoso es conocido como Tezcatlipoca ("el espejo que humea"), su color es el negro y el lugar donde reside, el norte. Su principal característica es la invisibilidad. Estaba relacionado con lo que significa la muerte, la maldad o la 
destrucción, ya que él era el gran brujo. El daba fama y riqueza pero también las quitaba. Dios creador de un complicado polimorfismo, representa originalmente al cielo nocturno que lo pone en relación con los dioses planetarios, con los dioses de la muerte, del frío, del pecado, de la miseria, del mal y la destrucción. Como dios de la muerte puede ser el equivalente necesario de Quetzalcóatl, el dios de la vida. Tezcatlipoca es la representación de la ansiedad humana y de la muerte.

Estas dos divinidades están en guerra desde los comienzos del mundo. Representan por su carácter positivo y negativo, el Eros y el Tanatos del lenguaje psicoanalítico. Desde otras perspectivas epistemológicas e históricas, nos recuerdan a Caín y Abel, o a Rómulo y Remo.

\section{El panteón nahua}

Además de los precedentes, otros habitantes del panteón vinculados con la muerte son Mictlantecutli (Señor del lugar de los muertos) y su esposa Mictecacihuatl (señora de los habitantes de la región de los muertos), residiendo en chicnauhmictlan, el lugar más profundo y subterráneo, correspondiente al noveno infierno. Ellos eran los que recibían los presentes que los difuntos llevaban consigo.

Por otra parte, la tierra con su capacidad germinadora y maternal se identifica con la mujer y su nombre es Coatlicue ("la que viste una falda de serpientes") divinidad aterradora, pues al tiempo que dispensa la vida, la retoma para descarnarla: "Mujer en su aspecto de madre, de guerrera, de verdugo. Autora de la vida y de la muerte, que acumula en su seno la ternura y el dolor" (3).

Estas divinidades no agotan el múltiple panteón náhuatl pero nos dan una idea de la religiosidad y de la importancia que para ellos tenía la muerte.

El hombre se explica a partir de la lucha de contrarios, de la muerte y resurrección que forman el mundo y en donde los dioses tienen un papel sobresaliente. $\mathrm{Y}$ el hombre los comprende porque ambos forman una unidad interdependiente en la que se apoyan el equilibrio y la continuidad del mundo.

El hombre náhuatl ubica a los dioses $\mathrm{y}$, una vez en su lugar, estos forman al hombre a partir de sus huesos ancestrales; sin embargo, para formarlos y darles vida es necesario el sacrificio de los dioses mismos. A su vez, el hombre es el responsable de sacrificarse por los dioses, de mantenerlos vivos y activos con su propia sangre. Dado que los dioses se sacrificaron e hicieron penitencia, recae en el hombre la obligación de ser el alimento de los dioses, so pena de que si no cumple correctamente su función, el orden será quebrado y las tinieblas y la confusión campearán en su mundo. Para que el sol siga su marcha hay que alimentarlo con el "agua preciosa", chalchihuatl, sangre de jade, sangre de los sacrificios que es garantía de preservación y mantenimiento del mundo.

\section{La caducidad de la existencia}

La caducidad de la existencia fue una reflexión a la cual se abocaron los tlamatinime (sabios) y los poetas o cantores: ¿Por qué el hombre es pasajero? ¿Por qué no permanece? ¿Por qué todo perece en la tierra? Preguntas que intentaban responder con sus versos:

\section{De la vida efimera:}

Sólo venimos a dormir. Sólo venimos a soñar:

No es verdad, no es verdad que venimos a vivir en la tierra.

En yerba de primavera venimos a convertirnos:

Llegan a reverdecer, llegan a abrir sus colores nuestros corazones,

Es una flor nuestro cuerpo: da algunas flores y se seca.

"No vivimos en nuestra casa

aquí en la tierra.

Así solamente por breve tiempo

La tomamos en préstamo.

¡Adornaos príncipes!

Solamente aquí

Nuestro corazón se alegra:

Por breve tiempo, amigos, estamos prestados unos a otros:

No es nuestra casa definitiva la tierra:

he aquí estas flores:

¡Adornaos, príncipes! (4)

De la angustia ante la muerte:

Me siento ebrio, lloro, sufro,

Cuando se, digo y recuerdo:

¡Ojalá nunca muriera yo,

ojalá jamás pereciera!

¿En dónde no hay muerte?

¿En dónde es la victoria?

Allá fuera yo...

¡Ojalá que nunca muriera yo, ojalá que jamás pereciera! (5) 
La muerte para estos personajes no tenía nada de espantable o de desdeñable, aprendieron a convivir con ella, supieron extraer lo paradójicamente extraño que la muerte posee: su vitalidad. Como en ningún pueblo su presencia se manifestó en sus mitos, se le encuentra como un ser vivo y actuante; constituyó un pilar de la ideología social y política; en el imaginario popular ciertas muertes eran deseadas, al ser motivos de prestigio humano y trascendencia divina; permeaba toda la actividad cultural de la poesía a la escultura, de la educación al juego, de la arquitectura al símbolo, de la religión a lo cotidiano, de la paz a la guerra, su búsqueda era inquieta y su llegada, serena. La muerte era, ciertamente no la nada, sino mas bien eso que transformaba, el "germen de la vida" (2): "La muerte y la vida no eran los extremos de una línea recta, sino dos puntos situados diametralmente en un círculo en movimiento. Así como toda vida conduce a la muerte, la muerte es la generadora de la vida" $(2,4)$.

\section{Los lugares para los muertos}

A aquéllos que morían a causa de algún fenómeno relacionado con el agua o por una tempestad, así como de ciertas enfermedades especiales se les asignaba el Tlalocan, la morada de Tláloc; los que dejaban de existir por cualquier otra enfermedad natural llegaban a la región de los muertos (Mictlán) (6). Había cuatro lugares a los cuales el fallecido podía dirigirse dependiendo del tipo de muerte que hubiera tenido.

A los muertos en el campo de batalla o en la piedra de los sacrificios (el techcatl), al ser capturados como prisioneros y luego sacrificados, se les otorgaba un gran reconocimiento: ellos no morían sino que trascendían, continuaban viviendo en el mejor de los lugares del más allá pues al morir iban hacia tonatiuhichan, la "Casa del Sol". Allí continuaban su actividad militar engrosando los batallones solares. Posteriormente, después de cuatro años se transformarían en colibríes y vivirían entre las flores.

Las mujeres muertas en parto eran equiparadas a guerreros. A la hora del parto le llamaban hora de la muerte. Eran las mocihuaquetzque que acompañaban al sol desde el "medio día" hasta el lugar donde era entregado al Mictlán para que realizara su recorrido por la región de los muertos. Esa parte occidental se llamaba cihuatlampa, la habitación de las mujeres.

Siendo también una sociedad agraria, tenían el ideal de una tierra fértil y generosa, abundante y placentera. Este lugar paradisiaco para los nahuas era el Tlalocan y se encontraba en el sur. A este lugar iban los elegidos por Tláloc, ya debido al premio que el dios da a quienes quiere, ya por el contagio con su fuerza, ya como un castigo que el señor de la lluvia envía a quienes lo ofenden (4-6). Los que iban allá eran los que matan los rayos o se ahogan, amén de los leprosos, bubosos y sarnosos, gotosos e hidrópicos.

A quienes no habían sido elegidos ni por Tonatiuh, ni por Tláloc, le aguardaba Mictlantecutli. A quien moría por finitud de su vida le esperaba el Mictlán, la región fría, sombría, cerrada de los muertos, ubicada al norte, donde después de difíciles pruebas y tras cuatro años de estar presente en el inframundo se diluiría.

Otra categoría de muertos que tenían trato especial, eran los lactantes. Ellos debían tener un lugar único para regresar pues no se habían contaminado, se encontraba en el décimotercer cielo, de donde habían venido. Ahí estaba el chichihuacuauhco, el árbol nodriza, del que mana leche y del cual los niños a su alrededor se alimentaban.

El culto de la muerte y la práctica del sacrificio son las características predominantes de la vida del pueblo náhuatl precolombino. Todo lo que vive está consagrado a la muerte y por lo tanto puede ser sacrificado prematuramente por el cuchillo de obsidiana (7).

Ninguna muerte es inútil. Todas conllevan una finalidad y una trascendencia, ninguna muerte es desdeñable ya que todas contienen un sentido.

Con la influencia del cristianismo se alteró la concepción nahua sobre la vida y la muerte. En un primer momento, para los indígenas después de la conquista, la vida era una tortura y la muerte una liberación.

\section{EI Día de los Muertos}

Resulta muy difícil dar cuenta de las múltiples expresiones mexicanas con que se celebra el Día de los Muertos. Cabe la reflexión sobre si más que celebrar a los muertos, los artífices de las ofrendas a los muertos, también llamados altares de muertos ¿no estarán celebrando a la vida? ¿No serán estos rituales una intensa prolongación de la vida en las inmediaciones de la muerte?

Aunque no se utilizaba en la época prehispánica, en la actualidad hay una flor que presta su estética a 
los altares, a las tumbas, a los caminos de las ánimas. Es la flor de innumerables pétalos, la flor infinita (cempasúchil, cempoalxóchitl, la flor veinte). Aunque no es la más bella, es la que ocupa un lugar privilegiado al recordar a los difuntos cada año.

A partir de la Conquista, las múltiples formas de celebración se concentraron en los días impuestos por la religión cristiana. Entre todas las ceremonias dedicadas a los muertos destacaban particularmente dos: la primera, celebrada en el noveno mes del calendario azteca, llamado Tlaxochimaco o Miccailhuitontli, es decir, fiesta pequeña de los muertos o fiesta de los muertos pequeños, y la otra, Xócotl Uetzi, también nombrada Hueymiccaihuitl, la fiesta grande de los muertos, festejada en el décimo mes. De este modo, con el advenimiento del cristianismo, se establecieron en México el primero y el dos de noviembre como los días de celebración de los difuntos, asimilando la tradición antigua, es decir primero la fiesta de los niños, y luego la de los adultos fallecidos.

La primera fiesta de los muertos, la dedicada a los muertos pequeños, llamada Miccailhuitontli en otros pueblos del Altiplano, era conocida por los mexicas como Tlaxochimaco, es decir "nacimiento de flores", ceremonia en la que "se ofrecían las primicias de las flores". Como se ha señalado, estos niños finados iban directamente al cielo más alto, es decir al lugar divino por excelencia, donde se reintegraban al magma vital del que nacía todo nuevo ser. Su muerte prematura no era una desgracia sino un sacrificio divino. En esta fiesta, las flores no se ofrecían a los pequeños muertos, sino que representaban a los niños mismos que se ofrecían al dios.

El mes siguiente, durante el Xócotl Uetzi, que significa la caída de xócotl, (un un árbol recién cortado y transformado en palo) estaba dedicado a los muertos adultos. Casualmente, las fiestas del mes Quecholli, en que se celebraban a los muertos en las guerras, coincidían en el siglo XVI, con las de Todos los Santos del calendario gregoriano, es decir, a principios de noviembre. Parecería que hubo allí una adecuación del ritual con el momento del año, quizás una adaptación tardía. En todo caso, el hecho de que al momento de la Conquista, la celebración nahua de los muertos adultos coincidiera con el ritual católico de Todos los Santos puede haber influido para dar a esta fiesta cristiana -secundaria en Europa- todo el fervor y el fasto con que se celebra desde entonces $(8,9)$.

Como hizo notar Paul Westheim (9): "Lo único que tiene en común el Día de los Muertos mexicano con la fiesta de los fieles difuntos, tal como se celebra en Europa, es el hecho de tratarse aquí y allá de un día consagrado a la memoria de los muertos queridos". Mientras que para el europeo la simple mención de la muerte es tabú, como si al rechazar el pensamiento se pudiera evitar el hecho, el mexicano se familiariza con la idea desde la niñez. Esta cercanía se manifiesta de muchas maneras, como por ejemplo en las múltiples expresiones eminentemente populares con que se dice que una persona murió: "se peló, se petateó, estiró la pata, lo sacaron con los tenis por delante, felpó, se difunteó, se enfrió, se ausentó, se nos fue, se lo chupó la bruja, se lo cargó patas de catre, entregó el equipo, acompañó a la flaca, dobló el pico, lo cafeteamos", etcétera.

La visita anual de los muertos no es ocasión de luto, sino motivo para una gran fiesta de celebración. Quizá una explicación para ello se encuentre en el Códice Matritense: "Decían los viejos: quien ha muerto, se ha vuelto dios. Decían: Se hizo dios, quiere decir que murió". Esto significa que el alma del difunto se torna en un ser sobrenatural con el poder de interceder por los familiares vivos. Finalmente vale la pena destacar una notable diferencia: "En la práctica católica ortodoxa, uno reza por las almas de los muertos para salvar su purgatorio. Los indígenas, por el contrario, no rezan por las almas, sino que les rezan a ellas". (10)

\section{CONCLUSIONES}

Las diferentes concepciones que en torno a la muerte y temas relacionados tuvo la histórica cultura Nahua reflejaron una mentalidad optimista, positiva de hondo contenido religioso y de esperanzas implícitas. Es particularmente impactante la cuidadosa clasificación de tipos de muerte, lugares de reposo de los fallecidos y destino final más allá de la vida terrena. Estas ideas, apenas modificadas por el advenimiento del cristianismo de la conquista española, prevalecieron en lo que podría ser una frase sumatoria de la epistemología básica de esta perspectiva: "Aprende a morir y aprenderás a vivir."

\section{Correspondencia}

Sergio J. Villaseñor-Bayardo

Universidad de Guadalajara

Av Juárez 976, Col Centro, CP 44100

Guadalajara, Jalisco

México

Correo electrónico: sergiovillasenor@gladet.org.mx 


\section{REFERENCIAS BIBLIOGRÁFICAS}

1. Sahagún B. Historia General de las cosas de la Nueva España. México: Editorial Porrúa; 1958.

2. Velasquez P. Códice Chimalpopoca: Anales de Cuauhtitlan y leyenda de los soles. México DF: Universidad Nacional Autónoma de México; 1992. p. 11.

3. Garibay AM. Historia de la literatura náhuatl. México DF: Universidad Nacional Autónoma de México; 1989.

4. Duverger C. La Flor letal. México: Fondo de Cultura Económica; 1993.

5. Garibay AM. Poesía Indígena de la altiplanicie. México: Universidad Nacional Autónoma de México; 1992. p. 135.

6. López Austin A. Cuerpo humano e ideología: Tamoanchan y Tlalocan. México DF: Universidad Nacional Autónoma de México; 1980
7. Matos E. Muerte a filo de obsidiana. México DF: Secretaria de Eduación Pública; 1986.

8. Lechuga R, Dufétel D, Gagnier M, Olmos G. Día de muertos, serenidad ritual. México DF: Artes de México;2002. p. 38-46; 62.

9. López G. Tesis de licenciatura en Filosofía. México DF. Universidad Intercontinental. 1996.

10. Lechuga R, Dufétel D, Gagnier M, Olmos G. Día de muertos, serenidad ritual. México DF: Artes de México; 2002. p. 33-36;62.
Recibido: 04/01/2013

Aceptado: 23/01/2013 